\title{
LA CONFORMACIÓN DE LA ANTROPOÉTICA A TRAVÉS DE LA TUTORÍA ACADÉMICA EN EDUCACIÓN SUPERIOR. (EL CASO DEL POSGRADO)
}

Víctor Manuel Alvarado Hernández
Docente en la Universidad Nacional Autónoma de México
México

Martin Manjarrez Betancourt

Docente en la Universidad del Valle de México

México

Recibido 5-V-2009 • Aceptado 5-VI-2009 • Corregido 17-VI-2009

Resumen: Actualmente hablar de violencia, guerra, terrorismo, cambios climáticos, daño ecológico y deterioro social, es hablar de una crisis generalizada de la humanidad. Con la Globalización ocurrió la pérdida de valores humanos, debido a la hegemonía del mercado, la tecnología y la informática. En la actualidad, se deben conciliar la materia y el espíritu, la naturaleza y la cultura, la ciencia y las humanidades, para propiciar un reencuentro entre la tecnología y el ser humano, integrando la memoria y el proyecto histórico, así como el futuro. En esto consiste el reto de la educación contemporánea; por ello, es necesario articular un nuevo paradigma para la educación: la antropoética. En este trabajo se expone el proceso de conformación de la antropoética, sus conceptos y las nociones de conformación, complejidad, tutoría académica y capital cultural. Se selecciona la estrategia institucional de la tutoría académica y el escenario de la educación superior, especificamente el posgrado, porque en ese nivel de la formación se generan diversas lineas de investigación y conocimiento que derivan en prácticas de aprendizaje para los demás niveles educativos.

Palabras clave: Antropoética, complejidad, tutoría académica, educación superior posgrado, formación humana.

\section{Introducción}

El daño ecológico, social y moral; sobrepoblación; la brecha cada vez más amplia y nítida de la desigualdad; el materialismo creciente. Con el falso conocimiento reduccionista de que conocer es la forma máxima para dominar, la asociación de ideas entre conocimiento y utilidad (Morin, 2005), han llevado a la idea del hombre jerárquicamente superior.

Todo ello ha contribuido a que pese sobre el hombre la amenaza de su autodestrucción. Todos esos poderes extraordinarios que tiene la humanidad de construirse a sí misma y su destino, la han llevado a caer en un desequilibrio, el hombre se incendia a sí mismo (González, 2009). Ante ese oscuro y tenebroso panorama, se ha tratado de aportar con el presente trabajo una forma que posibilite la realización del hombre con la educación. 


\begin{abstract}
Currently to speak of violence, war, terrorism, changes, ecological damage and social deterioration, is to speak of a generalized crisis of humanity. With Globalization came the loss of human values, due to the hegemony of the market, the technology and computer science. At the present times, the matter and the spirit, the nature and the culture, science and the humanities are due to conciliate, to cause an encounter between the technology and the human being, integrating the memory and the historical project, as well as the future. The challenge of the contemporary education consists on this; for that reason, it is necessary to articulate a new paradigm for education: the anthropoetic. In this work it is exposed the process of conformation of the anthropoetic, its concepts and the notions of conformation, complexity, academic tutorship and cultural capital. It is specifically selected the institutional strategy of the academic tutorship and a scenario of superior education, the post-degree one, because in that level of formation diverse lines of investigation and knowledge are generated that derive in practices of learning for the other educative levels.
\end{abstract}

Key words: Anthropoetic, complexity, academic tutoring, higher education (graduate), training humanization.
Es precisamente en este rubro de la educación donde se plasma la esperanza de salvaguardar la humanidad.

Para ello se conforma un marco conceptual donde se aborda categorías importantes como complejidad (humana e individual); comprensión; y antropoética. Categorías que servirán de apoyo para desarrollar y explicar como en la medida que la educación superior (posgrado) se enfoque a trabajar para la humanización de la humanidad ${ }^{1}$, efectuar la doble conversión de la educación; recibir educación para obedecer la vida, y para el día de mañana ser los orientadores y guías de la vida, lograr el reconocimiento de la unidad planetaria en la diversidad individual, la enseñanza del respeto al otro, a la vez, tanto la diferencia como la identidad consigo mismo, la enseñanza y desarrollo de la ética, en su triple aspecto, de la solidaridad, de la comprensión y de la ética del género humano (Morin, 2001).

Es así que desarrollando todos estos aspectos en sus diversas fases se tratará de consensuar que la conformación de la antropoética a través de la tutoría en el posgrado, es sumamente esencial, porque conlleva, entonces, a la esperanza de lograr la humanización de la humanidad, es decir, la esperanza de preservar y salvaguardar a la humanidad, a través de la educación, que le permita realizarse.

\section{Antropoética}

Hoy como antaño el mundo entero se agita en el odio y la violencia, la sociedad internacional se conmociono con los atentados, bombardeos, guerras civiles que convulsionan gran parte del mundo, como los bombardeos de Israel sobre la franja de Gaza o como el atentado realizado en un hotel de la cuidad turística de Bombay, en la India, donde irrumpió un comando terrorista en el restaurante del lujoso hotel, matando a casi la mayoría de comensales. Quizá lo no hubiera repercutido tanto en 
México de no ser por que una pareja que, turísticamente, visitaba aquel país se hospedaba en dicho hotel, ambos eran mexicanos. En diciembre 2008, un atentado narcoterrorista en la zona norte de México, llamó la atención porqué no sólo eran armas de alto calibre, sino que se utilizaron granadas fragmentarías. Ello ha causado aún más malestar, no sólo en México, sino en el mundo, por lo que el deterioro de la sociedad, no es un tema nacional, lo es planetario, ante ello los tratadistas opinan, como el Dr. José Manuel Estrada (1987), quien propone impactar un nuevo estado de consciencia sobre el planeta.

Algunos otros como Morin (2001) sugieren el retorno al humanismo, postulando que para evitar una tercera hecatombe en el planeta ${ }^{2}$ es necesario cambiar la conciencia, la condición humana, poner una nueva humanidad en marcha. Conformar la Antropoética, la cual debe considerarse como la conceptualiza el francés Morin (2001), una ética del bucle de los tres términos individuo $\leftrightarrow$ sociedad $\leftrightarrow$ especie, pues es de donde emerge la conciencia y nuestro espíritu propiamente humano, es una conciencia de la individualidad compleja más allá de la individualidad, que conlleva la esperanza de comprender la humanidad, para lograr la humanidad del sujeto como conciencia y ciudadanía planetaria, como toda ética es una aspiración y una voluntad, que al igual que todo conocimiento es una apuesta a lo incierto. Resultando así ser fundamental reflexionar no sólo en el tema de la violencia que por si sólo es cruda, sino también en la descomposición social y en el deterioro del medio ambiente.

\section{¿Cómo investigar sobre temas complejos como la sociedad y el individuo?}

Reflexionar sobre estos tópicos, evitando caer en reduccionismos, estructuras, en lugares comunes, en pensamientos simples, soluciones mágicas o simples especulaciones, lo hacen ser algo más complejo ${ }^{3}$ una tarea ardua y extremadamente difícil. Para empezar, sugerimos analizar que el individuo es un sujeto sumamente complejo y que al ser productor de la sociedad la hace ser compleja, lo que encierra la complejidad de la especie.

\section{¿Qué le hace ser al hombre un sujeto complejo?}

Tratar de describirlo es una tarea que requiere un espacio considerablemente amplio, por tal motivo, sólo se mencionara que su complejidad se basa en su naturaleza, que no es el simple ser biológico conceptualizado en el paradigma cartesiano, que también lo conforma una esencia antropológica, psíquica, ecológica, sociológica, que tiene objetividades y a la vez es subjetivo, que es un ser racional y a la vez tiene magia y mitos. Por ende las sociedades humanas, tal como ocurre en los individuos, atraviesa por etapas que suelen ser cruentas, depredativas, llegando a tomar matices dolorosos. Momentos que llegan a caracterizarse por expresiones exaltadas de violencia y barbarie. No es nada difícil encontrar muchos ejemplos históricos en países o regiones, como los que enunciamos al inicio, en los cuales pareciese que efectivamente el único calificativo válido para el ser humano es la de bestia infame y alevosa.

Frente a un escenario caracterizado por la barbarie surge la pregunta de fondo ¿Qué hacer? No dudamos que ante la complejidad del problema y la complejidad citada del sujeto, existirán diversas teorías y posibilidades de responder a tal cuestionamiento. Habrá quienes asumirán la posición pesimista-miedosa, pensando que el ser humano es un depredador natural que solo vigilándolo y castigándolo ejemplarmente, es posible controlarlo (Foucault, 2004).

Con este trabajo se pretende establecer un acercamiento a las cegueras del conocimiento (Morin, 2005) para aclarar que el hombre no es simplemente un ser biológico, sino que es un ser síquico, 
antropológico, sociológico, ecológico, con un pensamiento y un comportamiento sumamente complejos, y si se concientiza sobre su complejidad, es fácil darse cuenta que el origen de muchos de los problemas humanos sigue, aligualque en la antigüedad, en el mundo occidental $u$ oriental, siendo el mismo...LA EDUCACIÓN ${ }^{4}$.

Bajo la influencia del método cartesiano, se ha producido la inteligencia ciega, (Morin, 2005) conduciendo a construir una sociedad basada en la educación cuyo objeto básico es formar al individuo en el manejo tener-hacer (Damm, 1989) la ceguera del conocimiento ha producido que la educación se enfoque en un sistema funcionalista que instruye hombres aptos a contribuir con el sistema económico (sea capitalista, liberalista, o neoliberalista) convirtiendo al individuo en un tilde productor que puede conducirse a cualquier parte menos a su realización, por que ha perdido su libertad de decidir-se (Damm, 1989) renunciando a vivir una vida plenamente humana.

El hombre aumento su cantidad de vida, se masifico y cosifico, ha querido vivir mejor, sin ser mejor, renuncio a los horizontes cualitativos por los cuantitativos, se ha limitado a la posesión de bienes materiales (Damm, 1989). Este cause educativo es una consecuencia lógica de la denominada sociedad de consumo (Chomsky, 2004) en la cual el ser humano se valora por su capacidad de consumir, así cuanto mayor sea su consumismo será más alta su autoestima.

Tener los mejores y más lujosos autos, lujosa casas, comodidades, es sinónimo de éxito social, lo que en el pobre es un defecto, en el rico es una virtud. Sin embargo, estas posibilidades del ser humano contemporáneo no le otorgan el verdadero ejercicio de la libertad óntica (libertad de ser), la cual, se ha conducido a esclavizar al hombre al bien-tener y al bien-hacer, que lo lleva al bien-estar, olvidando el bien-ser.

La tendencia de búsqueda frenética por el tener ha llevado a afianzar una sociedad fuertemente inequitativa, en la cual unos pocos tienen mucho y otros tienen muy poco, provocando un caldo de cultivo de problemas bastante complejos para la humanidad, tales como la delincuencia, la corrupción, la violencia, etc. Siendo urgente abordar la educación bajo un componente que hasta ahora, creemos poco tratado: una consciencia de la concepción compleja del género humano, una ética propiamente humana, es decir, una ANTROPOÉTICA ${ }^{5}$.

La antropoética debe considerarse como una ética de la consciencia de la conceptualización compleja del género humano que comprende la triada individuo $\leftrightarrow$ sociedad especie, $\boldsymbol{\leftrightarrow}$ donde las interacciones entre cada uno de ellos los hace sostenerse, retroalimentarse y religarse. Cada uno de estos términos es a la vez co-productor de ser humano es a la vez medio y fin de los otros. Toda concepción del género humano significa el desarrollo conjunto de las autonomías individuales, de las participaciones comunitarias y del sentido de pertenencia a la especie humana.

La educación del futuro, desde este punto de vista, deberá considerar la antropoética, es decir la ética de la triada individuo $\leftrightarrow$ sociedad $\leftrightarrow$ especie, de donde emergen nuestra conciencia y nuestro espíritu propiamente humano, como lo expone el tratadista Morin (2001), el cual se ha venido mencionando. La antropoética no pide una espiritualidad o religiosidad especifica, puesto que se puede ser espiritual y no religioso o ser muy religioso y no espiritual, sino una ética que permita al ser humano su autonomía individual, pero a la vez, su participación comunitaria, su sentido de pertenencia a la especie humana, lo que le permita descubrir que en el cosmos hay un orden pero también un desorden. Andar en el camino sinuoso, incierto y riesgoso del conocimiento complejo, que lo lleve a valorar su interior, su entorno y su contexto, a tomar conciencia de su ser físico, pero también biológico, antropológico, síquico, ecológico, sociológico, etc., que lo hace ser complejo y por decisión, asumir la responsabilidad sobre sí-mismo, sobre su planeta, sobre su cosmos, trabajando para 
conocerlos y entenderlos, pero con la certeza de que al final del camino del conocimiento es incierto e infinito, lo que lo hace seguir conociendo, avanzando, desarrollando habilidades y estrategias para asumir el riesgo del conocimiento complejo.

La solución de sus problemas complejos, como la delincuencia, terrorismo, corrupción, violencia, deterioro ecológico, etc., no se consigue bajando la cabeza o aislándose con la esperanza de que todo se resuelva solo o por intervención divina.

Ante el estado de barbariedad del presente y su particular complejidad se le de un giro a la educación, que se trabaje para acelerar la práctica de una educación basada en la antropoética. La cual se fundamenta, como ya se menciono, en una vivencia ética de la triada individuo sociedad $\leftrightarrow$ especie $\leftrightarrow$ y no simplemente moralista, una ética de la humanidad, una ética del discernimiento, del intelecto, del conocimiento de lo complejo, como capacidad para evitar tanto odio, rencor, violencia, tanta basura que sea foco de antivalores que deshumanizan al ser humano; la ética de la austeridad que evita comprar y comprar simplemente por imposición social (por la burla de traer una ropa fuera de moda, un carro que consideran carcacha, un celular pasado, instrumentos que quizás si sean pasados pero nos sirven para vestirnos, transportarnos o comunicarnos) y no por necesidad (Bourdieu, 1999).

Lo anterior requiere una ética del estudio donde se comprenda que no hay absolutos, que el conocimiento es tan complejo que tiene riesgos e incertidumbres, más no por ello se deba dejar de aprender, de investigar, sino desarrollar estrategias educativas para modificar e innovar, para conocer, entender y comprender las antinomias, las antípodas, el orden y el caos que existe en el cosmos y que no pueden separarse por que uno es sustento del otro, entender a la naturaleza, al ecosistema, comprender las disfuncionalidades y no caer en la ceguera, en el engaño, se trata de acercarse a la realidad desde la multiplicidad y diversidad de análisis que posibilitan el pensamiento complejo (Mannheim, 1987), la ética del gusto existencial por la vida y con la vida, el respeto y el valor por ella, no sólo por el hecho de estar vivos, sino aprender a respetarla y valorarla (Latapi, 2002).

Pero ¿Cómo lograr educar en la antropoética? Para ello es necesario voltear la mirada hacia las estructuras, programas e instituciones educativas que son las que formaran a los docentes, investigadores y tutores venideros, que a su vez formaran a las futuras generaciones (Latapi, 2004).

Más luego entonces ¿hacía dónde dirigir la mirada, hacía la educación básica, hacía la media-superior, hacía la superior o bien hacía el posgrado?

Una posible respuesta se encuentra en las ideas propuestas por Ruiz, Argueta, Martínez, Corrales y Hernández (2006) ${ }^{6}$, quien afirma, que en México se requiere un posgrado más fuerte $\mathrm{y}$, al mismo tiempo, con una calidad más homogénea, con garantía de excelencia, pues no puede pensarse elevar la calidad en la educación básica o media superior, sino se tiene un posgrado sólido, donde se forme a los docentes que impartirán clases en el resto de los niveles educativos.

Así recientes investigaciones se han enfocado en el posgrado, por cuanto esté nivel de estudios cobra gran importancia porque forma recursos humanos del nivel más alto que puede tener un país. Siendo este nivel donde se forman los futuros enseñantes de los otros niveles educativos, por lo que retomando esta idea de que se necesita un posgrado sólido y de calidad para incidir en los otros niveles educativos, estimamos necesario irrumpir aquí, para dar ese cambio que la educación necesita, educando en la antropoética, para así atender, lo que González (2009) denomina la crisis del humanismo que hemos venido padeciendo en los últimos tiempos, debido a la demasía, el exceso y la soberbia. Contemplamos la antropoética en la formación de los alumnos de posgrado, con 
el objetivo de que comprendan que el conocimiento no es para dominar a la naturaleza, ni mucho menos al hombre, la asociación entre conocimiento y utilidad debe desecharse, pues la antropoética evidencia que el conocimiento no es para dominar la naturaleza ni obtener la mayor explotación del hombre, sino que el conocimiento hace posible desentrañar la particular contingencia del universo.

Se considera que la conformación de la antropoética permitirá a los alumnos de posgrado asumir los riesgos, enfrentar las incertidumbres que depara el conocimiento complejo. Se contempla educar en la comprensión del conocimiento complejo, para que los alumnos del posgrado, aprendan que la naturaleza no es un objeto para apropiárselo y sacarle provecho (utilidad), que es su entorno, su hábitat, por lo que destruirla, es destruirse a sí mismos, enseñarles que deben asociar el conocimiento con la innovación, la creatividad, la modificación, la generación de un pensamiento crítico que les permita la construcción de nuevos paradigmas o la discusión de los actuales, que permita un desarrollo económico y social, más justo, más equitativo.

\section{¿Qué cualidades requieren los alumnos de posgrado para la conformación de la antropoética?}

Una educación para la conformación de la antropoética requiere de la decisión consciente y clara de los alumnos del posgrado de que:

\footnotetext{
* Asumir la humana condición individuo $\leftrightarrow$ sociedad $\leftrightarrow$ especie en la complejidad de nuestra era.

* Lograr la humanidad en ellos mismos en su conciencia personal.

* Asumir el destino humano en sus antinomias y plenitud.
}

Pero ¿Qué comprende en sí la antropoética en los alumnos del posgrado?
El aporte de la antropoética hacia la concepción de una nueva manera de ver la educación, se sintetiza en los siguientes puntos:

- Trabajar para la humanización de la humanidad.

- $\quad$ Efectuar el doble pilotaje del planeta; es decir los alumnos de posgrado deben obedecer la vida y a la vez guiarla.

- Lograr la unidad planetaria en la diversidad; los alumnos del posgrado comprenderán que las fronteras y limitaciones de los territorios, no están en la geografía, sino sobre el mapa.

- Respetar en el otro, a la vez, tanto la diferencia como la identidad consigo mismo.

- Desarrollar la ética de la solidaridad. - Desarrollar la ética de la comprensión.

- Para posteriormente ellos, como futuros docentes, investigadores, tutores, enseñen la ética del género humano.

Ahora bien ¿Cómo transmitir, cómo enseñar, cómo reunir todos esos elementos y disposiciones para la conformación de la antropoética en los alumnos del posgrado?

El conflicto magisterial, que actualmente existe en México ha dejado entrever la crisis de las instituciones, específicamente, la crisis de las instituciones educativas, ante la descomposición del tejido social, la crisis de las instituciones educativas es un signo más del deterioro social, apareciendo en el fondo del conflicto magisterial un hecho que no se ha vislumbrado, que nadie cuestiona:

¿Al entrar la humanidad en crisis, arrastra con ella sus instituciones educativas, por ser la educación un producto cultural y la cultura en sentido genérico, un producto de las interacciones de la triada: individuo $\leftrightarrow$ sociedad $\leftrightarrow$ especie? O bien ¿Son las políticas educativas el origen y causa de todos los problemas de la humanidad?

Estas son palabras mayores, son algo fuerte pero que sin duda alguna, cuestionan 
la viabilidad de la escuela. Como todos los axiomas cartesianos, la escuela se presenta como un paradigma, en el cual se afirma que ninguna persona, sea niño, joven o adulto, puede aprender sin dosis de enseñanza escolarizada y, en consecuencia como dicen y pretenden hacer creer a las autoridades gubernamentales en materia de educación en México, que es necesario reformar la calidad de esta enseñanza escolarizada (Olmos, 2008). ¿Será tan simple el problema de la humanidad, del país, que con una simple reforma a la enseñanza escolarizada bastará para solucionar sus problemas tan complejos? ¿o será una ceguera más que no los deja ver la complejidad de la humanidad y de sus problemas?

Considerando la historia, antes del siglo XVII, la humanidad no necesitó de dosis de enseñanza escolarizada para sobrevivir y crear cultura, ya que la escuela surgió en el siglo XVII como una propuesta de Comenius (2005). Así, en este sentido, habría que agregar que si la educación ha entrado en crisis no es por que la enseñanza de los profesores sea deficiente, ni mucho menos por su escasa actualización, por lo que consideramos no hay tal necesidad de obligarlos, sobre todo al sector magisterial recalcitrante a regresar a las aulas, sino por que las autoridades con sus políticas educativas, se han apartado de los postulados de Comenius, se ha dejado de servir a los fines de su creación histórica. En el caso de México se ha hecho caso omiso a las sugerencias de Morín (2001), quien nos dice los saberes que deberá contemplar la educación del futuro, por lo que las políticas en materia educativa sólo se han politizado entre los gremios sindicales de los trabajadores de la educación y las autoridades gubernativas, sin tomar ninguna medida pertinente para enfrentar la crisis en la educación. Así la Institución de la escuela, sugerida por Comenius, en México ha dejado de cumplir con los postulados de éste autor, por lo que no es regresar al magisterio a las aulas, ni atender sólo la crisis escolar, sino comprender que la crisis de la humanidad arrastra consigo la de sus instituciones, por lo tanto se debe atacar el problema redireccionando la educación hacía la conformación de la antropoética.

Continuando con la idea de que la escuela ha entrado también en crisis, Illich (2004) ha mostrado a la escuela como una estructura injusta de segregación social. Donde la escuela se convirtió en el monopolio absoluto de la enseñanza para acceder al poder, es decir, a los puestos profesionales. Ha generado a lo largo de su existencia una parálisis de la capacidad innata del hombre de aprender.

Las políticas educativas han sido tendenciosas, han criminalizado el aprendizaje independiente, al que se da en el seno de la familia, del hogar, ya sea, con los padres, con los hermanos mayores, o con algún pariente o conocido de la comunidad, quienes por su capital cultural (Bourdieu, 2000), seducen, convirtiéndose en tutores. Se ha afirmado por decreto que nadie puede saber sino no posee un papel certificado, provocando un subdesarrollo en la confianza del hombre en aprender por sí mismo a través de lo que la familia, la comunidad, el tutor, le da y necesita. La competencia en la carrera del aprendizaje la han hecho, además de cruenta y cruel, más segregativa, pues acceder al saber y, luego, al trabajo, en medio de una sociedad más demandante de escolaridad, la educación especializada o del posgrado, deja de ser una posibilidad para todos, aunado a que los empleos profesionales están saturados, la educación profesional es para unos cuantos, que de antemano se someten a la dosis de calidad que las autoridades gubernativas han determinado, generando así una nueva clase de pobres y segregados.

Para atender esta crisis escolar, como ya se dijo, es necesario solventar la crisis de la humanidad, por ello se plantea que la tutoría académica ${ }^{7}$. Surge así un nuevo cuestionamiento ¿Qué es la tutoría académica? es el vehículo que conducirá hacia la conformación de la antropoética, 
¿en que consiste la tutoría académica? El Diccionario de las ciencias de la educación (Sánchez, 1999) la conceptualiza: Una orientación, a lo largo de todo el sistema educativo, para que el alumno se supere en rendimientos académicos, solucione sus dificultades escolares y consiga hábitos de trabajo y estudio, de reflexión y de convivencia social que garanticen el uso adecuado de la libertad responsable y participada. Para Latapi (1988) es una modalidad de instrucción en la que un maestro (tutor) proporciona educación personalizada a un alumno o a un grupo reducido. Generalmente se adopta como una medida emergente o complementaria para estudiantes con dificultades para seguir los cursos convencionales. Como se desprende de dichas definiciones contienen términos ya rebasados, por lo que para efectos de este artículo entenderemos la tutoría académica como el proceso de acompañamiento en la formación de lo alumnos del posgrado, mediante la atención personalizada a un alumno o a un grupo de alumnos por tutores formados para esta modalidad, con el objetivo de educar en la conformación de la antropoética para aprender la complejidad tanto del pensamiento como del sujeto mismo, impulsando el cambio del paradigma de la enseñanza al paradigma del aprendizaje. Así como ya se dijo, la tutoría académica será el vehículo por el cual se aprenda la antropoética, para humanizar a la humanidad, darle una formación humanitaria al alumno del posgrado, para que posteriormente él que va a ser el posible tutor de muchos otros alumnos de los diversos niveles, la aprenda, la guie y la difunda, pero para ello debe romper con los paradigmas tradicionales escolarizados y enfatizar más en el aprendizaje que en la enseñanza, es decir, que su finalidad no será transmitir conocimientos sino orientar de forma individualizada para la formación humana del alumnado. El rol del tutor más que instruir es formar, centrándose en enseñar para comprender la complejidad humana.

\section{Conclusiones}

El sistema tutorial en los programas de posgrado, estimamos debe abordarse desde una concepción compleja del género humano. Es decir, desde una ética propiamente humana. Por ello creemos que una de las estrategias institucionales idóneas para alcanzar los objetivos de la educación y llegar a la construcción de estudios serios sobre la conformación antropoética de los alumnos de posgrado, es la TUTORÍA. Donde es posible trabajar la voluntad de aprender. Es por ello que en el tutor se contempla una manera de alcanzar la formación de cada uno de los alumnos del posgrado, es decir, una conciencia individual más allá de su individualidad.

Si el aprender, como dice Sicilia (2008) significa obtener una nueva habilidad o un nuevo entendimiento, vislumbramos al tutor como la estrategia en el campo educativo para que se de ese nuevo entendimiento de la ética humana y se construya la conformación antropoética en los alumnos de posgrado.

La antropoética como un nuevo entendimiento que conlleva esperanza, la esperanza de lograr la humanidad como conciencia y ciudadanía planetaria, que comprenda a la vez, como toda ética, una aspiración y una voluntad, hacía un país mejor, hacía un planeta cada vez mejor, sin olvidar que ello en si es una apuesta a lo incierto.

\section{Notas}

1 Esto que pareciera una redundancia, es un problema antropohistórico, donde el hombre a pesar de su evolución del homínido al homo sapiens, no ha asumido su humana condición, es por ello que se sugiere una educación para la comprensión humana, una educación para conocer los obstáculos a la comprensión (externos: ruido, polisemia, entre otros, e internos: egocentrismo, etnocentrismo, espíritu reductor). Ver Morin (2001, pp. 87-98).

2 Las dos Hecatombes a que nos referimos son los conflictos armados más grandes y sangrientos de la historia mundial que fueron la 
$1^{\mathrm{a}}$ y $2^{\mathrm{a}}$ guerras mundiales (1914 a 1918 y 1939 a 1945 , respectivamente).

Literalmente, Complejidad (Complexus) significa lo que está tejido junto. Por lo tanto, hay complejidad cuando los diferentes elementos que constituyen un todo (económico, político, sociológico, sicológico, afectivo, mitológico) son inseparables y existe un tejido interdependiente, interactivo, e interretroactivo entre el objeto de conocimiento y su contexto, las partes y el todo, el todo y las partes entre ellas, es la unión entre la unidad y la multiplicidad (cfr. Morin, 2001, pp. 35-38).

El espíritu reduccionista ha traído como consecuencias las cegueras del conocimiento, dogma cartesiano que ha seguido la educación. Ello aunado a que no es lo mismo educar para las matemáticas que educar para la comprensión humana (cfr. Morin, 2001, pp. 89-95).

Como lo hemos dicho si el conocimiento es complejo, debemos educar para comprender lo complejo, aún y cuando el sujeto y el objeto son complejos, también lo hemos mencionado aquí, que no es lo mismo educar para enseñar las matemáticas que para comprender la complejidad humana, por ello se sugiere que sea la Antropoética la que nos permita la comprensión humana (cfr. Morin, 2001).

Quien fuera la secretaria de Desarrollo Institucional de nuestra máxima casa de estudios (UNAM).

Para efectos de este artículo entenderemos la tutoría académica como el proceso de acompañamiento en la formación de los alumnos del posgrado, mediante la atención personalizada a un alumno o a un grupo de alumnos por tutores formados para esta modalidad que eduquen en la comprensión y conformación de la antropoética, impulsando el cambio del paradigma de la enseñanza al paradigma del aprendizaje.

\section{Referencias bibliográficas}

Bourdieu, P. (1999). Razones prácticas, sobre la teoría de la acción. Barcelona: Anagrama.

Bourdieu, P. (2000). Capital cultural, escuela y espacio social. México: Siglo XXI.
Chomsky, N. (2004). El nuevo orden mundial (y el viejo). Barcelona: Crítica.

Comenius, J. (2005). Didáctica magna. México: Porrúa.

Damm, A. (1989). Libertad: Esencia y existencia. México: Minos III Milenio Editores.

Estrada, J. (1987). Libertad responsable y educación, Libertad de enseñanza. Buenos Aires: Nueva Visión.

Foucault, (2004). Vigilar y castigar: Nacimiento de la prisión. México: Siglo XXI.

González, J. (2009, diciembre). Necesario, atender la crisis del humanismo. Gaceta UNAM, 3860, 10.

Illich, I. (2004). La sociedad desescolarizada. México: FCE.

Latapi, P. (1988). La enseñanza tutorial: Elementos para una propuesta orientada a elevar la calidad. Revista de la Educación Superior-ANUIES, 68, 5-19.

Latapi, P. (2002). La moral regresa a la escuela. México: Universidad Nacional Autónoma de México.

Latapi, P. (2004). El debate sobre los valores en la escuela mexicana. México: FCE.

Mannheim, K. (1987) Ideología y utopia. Introducción a la sociología del conocimiento. México: FCE.

Morin, E. (2001). Los siete saberes necesarios para una educación del futuro. México: UNESCO.

Morin, E. (2005). Introducción al pensamiento complejo. Barcelona: Gedisa. 
Olmos, G. (2008). Alianza por la simulación. Revista Proceso, 1668, 6-12.

Ruiz, R., Argueta, A., Martínez, V., Corrales, V. y Hernández, B. (2006). Los estudios de posgrado en México: Diagnóstico y perspectivas. México: UNESCO-IESALC.
Sánchez, S. (1999). Diccionario de las ciencias de la educación. Madrid: Aula Santillana.

Sicilia, J. (2008, 19 de ocgtubre), La escuela en crisis. Revista Proceso, 1668, 50. 